NBER WORKING PAPER SERIES

ESTIMATING THE CONTINUOUS TIME CONSUMPTION BASED ASSET PRICING MODEL

\author{
Sanford J. Grossman \\ Angelo Melino \\ Robert d. Shiller
}

Working Paper No. 1643
NATIONAL. BUIREAUI OF ECONOMIC RESEARCH
1050 Massachusetts Avenue
Cambridge, MA 02138
June 1985

The research reported here is part of the NRER's research program in Financial Markets and Monetary Economics. Any opinions expressed are those of the authors and not those of the National Bureall of Economic Research. 
NBER WORKING PAPER \#1643

June 1985

\section{Estimating the Continuous Time \\ Consumption Based Asset Pricing Model}

\section{ABSTRACT}

The consumption based asset pricing model predicts that excess yields are determined in a fairly simple way by the market's degree of relative risk aversion and by the pattern of covariances between per capita consumption growth and asset returns. Estimation and testing is complicated by the fact that the model's predictions relate to the instantaneous flow of consumption and point-in-time asset values, but only data on the integral or unit average of the consumption flow is available. In our paper, we show how to estimate the parameters of interest consistently from the available data by maximum likelihood. We estimate the market's degree of relative risk aversion and the instantaneous covariances of asset yields and consumption using six different data sets. We also test the model's overidentifying restrictions.

Sanford J. Grossman Department of Economics Dickinson Hall Princeton University Princeton, N.J. 08544 (609) 452-4035
Angelo Melino Department of Economics University of Toronto 150 St. George Street Toronto, Canada M5S 1Al (416) 978-6541
Robert J. Shiller

Cowles Foundation for Research in Economics Yale University Box 2125 Yale Station New Haven CT 06520 (203) 432-4134 


\section{Introduction}

In this paper, we provide an empirical test of the continuous time intertemporal capital asset pricing model, first proposed by Merton[1971]. The model as clarified by Breeden[1979] implies that ari asset will be priced so that the expected return required will increase with its covariability with per capita consumption growth. Frevious tests of this theory (e.g. Grossman-Shiller[1980], Hansen-Singleton[1983]) have examined discrete time versions of the model under the assumption that the timing interval of the model matches exactly the sampling interval for available data on per capita consumption. That is, if we have data on quarterly consumption, then the time period is assumed to be 1-quarter of a year. We show that if the true model is a continuous time model, and time averaged data (such as quarterly consumption) is used to test it, then substantial biases may be introduced unless the estimation procedure is corrected to take account of the effects of time averaging. We provide a procedure for obtaining consistent estimates with time averaged data. We ther estimate and test the model using data on per capita consumption and the cumulated real returns to holding portfolios of stock:s, bonds, and short-term paper.

I The Model

It is useful to review the Merton model. Our discussion follows closely the exposition of its generalization in Grossman-Shiller[1982]. In a discrete time model, each consumer is assumed to maximize a time-additive utility function over a single consumption good 
(2. 1)

$$
U=\sum_{j=0}^{T / h} g^{h j} u(c(j)) h
$$

where $T$ is his time horizon, $c(t)$ is consumption at time $t$ arid

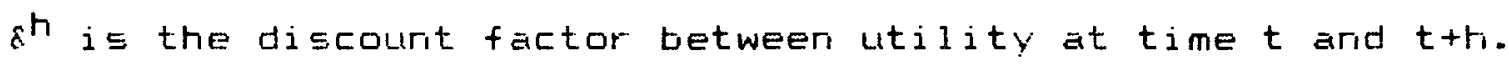
For the purposes of this paper, we assume that the period utility function is of the constant relative rist aversion (or isoelastic) form

$$
\text { (2.2) } \quad u(c)=c^{1-A} /(1-A) .
$$

Let $v_{i}(t)$ denote the value of asset $i$ at $t$ ime $t$ including any accrued cash disbursements (such as dividends or coupons) earned between $t-h$ and $t$. Assume that asset i is freely tradeable. A standard argument shows that

$$
(2.3)
$$

$$
E_{t} \varepsilon^{h} u^{\prime}(c(t+h)) v_{i}(t+h)=u^{\prime}(c(t)) v_{i}(t)
$$

where the expectation is conditioned on all the information possessed by the trader at time $t$. Using ( 2.2 ) and iterating $(2.5)$, we can write

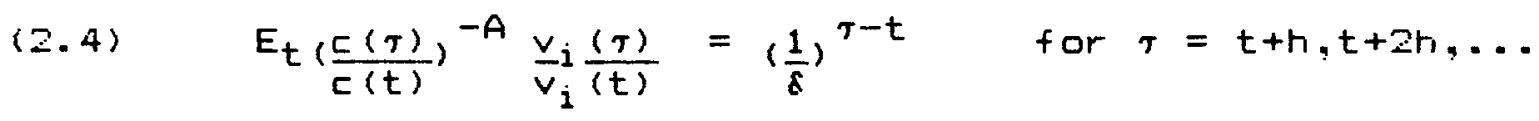

If we take the limit to continuous time and apply Ito's Lemma, we obtain

$$
\text { (2.5) }
$$

$$
\begin{aligned}
E_{t} \frac{d v_{i}}{v_{i}}+\frac{1}{2} * A(A+1) * \operatorname{Var}\left(\frac{d c}{c}\right) & -A * E_{t} \frac{d c}{c}+1 n \delta d t \\
& =A * \operatorname{Cov}\left(\frac{d c}{c}, \frac{d v_{i}}{v_{i}}\right)
\end{aligned}
$$

where Var and Cov denote the variance and covariance operators. Note that (2.5) holds for an individual. Under various assumptions about heterogeneity of information and wealth, (2.5) 
can be aggregated over individuals so that $c$ can be interpreted as per capita consumption and $A$ is replaced by a particular weighted average of the individual consumer's A (see Grossman-Shiller[1982]). Clearly (2.5) holds for all tradeable assets. If $F_{i}^{e}$ is defined as the excess rate of return of asset i over say short-term paper, then (2.5) can be used for these two assets to yielo

$$
\text { (2.6) } \quad E F_{i}^{e}=A * \operatorname{Cov}\left(F_{i}^{e}, d c / C\right) \text {. }
$$

The aggregate parameter of relative rist: aversion can be computed by (2.6) given data on mean excess returns and the covariances between excess returns and per capita consumption growth. Table 1 provides some estimates of $A$ based on the descriptive statistics from Table $\Xi$. The various data sets and variable definitions are described more fully in Section III. At this point, we simply wish to draw attention to one of the important empirical anomalies associated with the model and the potential role for time averaging as an explanation. The table shows that the mean excess return on stocks is associated with a relatively small covariance with consumption changes. Therefore this can be justified only by an implausibly high estimate of the rist: aversion parameter. Similiar conclusions are reached by examining the excess returns on bonds.

One explanation for this is based on the idea that a time averaged variable is smoother than the same point sampled variable. In particular, if the true model holds in continuous time then the instantarieous rates of change in consumption can be more 


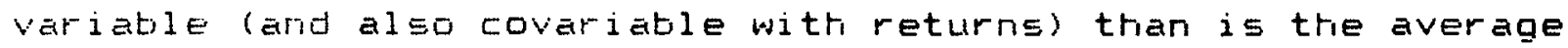
consumption change across years or quarters.

\section{An Example}

To understand this effect, consider the following very simple process for $v_{i}^{e}$, the value of asset $i$ in excess of asset 1, and consumption:

$$
\begin{aligned}
& \text { (2.7a) } d c=p d t+d E \\
& \text { (2.7t) } d v_{i}^{e}=\mu_{i} d t+d n_{i}
\end{aligned}
$$

where $\equiv, n_{i}$ are correlated Erownian motions with Cov $\left(d E, d n_{i}\right)=\sigma_{i} d t$. Let $\bar{c}(t)$ and $\bar{v}_{i}^{e}(t)$ be the time averaged values of $c(t)$ and $v_{i}^{e}(t), i . e$.

$$
\bar{c}(t)=T^{-1} \int_{O}^{T} c(t+5) d s \quad \bar{v}_{i}^{e}=T^{-1} S_{O}^{T} v_{i}^{e}(t+s) d s .
$$

We will show that

$$
\text { (2.B) } \begin{aligned}
\operatorname{Cov}\left(\bar{c}, \bar{v}_{i}^{e}\right) & \equiv E\left[(\bar{E}(t)-\bar{E}(t-T))\left(\bar{v}_{i}^{e}(t)-\bar{v}_{i}^{e}(t-T)\right)\right]-f T \mu_{i} T \\
& =2 / 3 T \sigma_{i} .
\end{aligned}
$$

If we normalize $T=1$, then the covariance of time averaged consumptions changes and price changes is $2 / 3$ of the instantaneous value $\sigma_{i}$. Foughly speating, this would lead us to overestimate A by $50 \%$.

To uniderstand (2.8) just note that

$$
\begin{aligned}
& \text { (2.8) } \bar{c}(t)-\bar{E}(t-T)=S_{0}^{T} \text { pds }+T^{-1} S_{0}^{T} S_{t+s-T}^{t+s} d E(\tau) d s \\
& =p T+T^{-1} S_{0}^{T} S_{t+s-T}^{t} d E(T) d s \\
& +T^{-1} S_{0}^{T} s_{t}^{t+s} d \in(\tau) d s \\
& =\rho T+T^{-1} s_{t}^{-t}-T(T-t+s) d E(s) \\
& +T^{-1} \int_{t}^{t+T}(t+T-s) d E(s) \text {. }
\end{aligned}
$$


A similiar expression may be derived for $\bar{v}_{i}^{e}(t)-\bar{v}_{i}^{e}(t-T)$. Hence, $(2.10) E\left[(\bar{C}(t)-\bar{E}(t-T))\left(\bar{v}_{i}^{e}(t)-\bar{v}_{i}^{e}(t-T)\right)\right]$

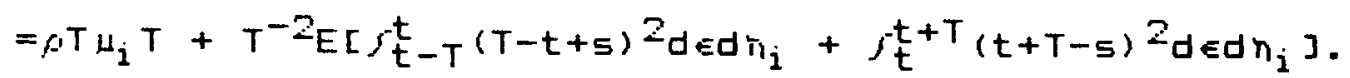

Equation (2.8) is easily derived from the last expression.

The purpose of this example is to give the reader a relatively simple view of the effect of time averaging in generating a stochastic process which is "smoother" than the instantaneous process. This suggests the possibility that assets appear to have a low rist: (i.e. low covariance with consumptior changes) because measured consumption changes are less variable than instantaneous consumption changes. Since it is the covariance with instantaneous consumption changes that is the relevant measure of an asset's risk, this leads us to overestimate $A$. In our simple example, A is overestimated by $50 \%$. As we shall sef below, for certain processes, the bias can be arbitrarily large.

Multivariate Model

In our empirical worl:, we postulate a slightly more complicated stochastic process for consumption and asset values. Define $Y(t)$ according to

$$
Y(t)=\left[\begin{array}{l}
1 n_{1}(t)-v_{c}-g_{c} t \\
1 n v_{1}(t)-v_{1}-g_{1} t \\
\ln v_{2}(t)-v_{2}-g_{2} \\
1 n v_{3}(t)-v_{3}-g_{3}
\end{array}\right] \equiv\left[\begin{array}{c}
c(t) \\
v_{1}(t) \\
v_{2}(t) \\
v_{\Xi}(t)
\end{array}\right] .
$$

We assume that $Y(t)$ satisfies the stochastic differential equation

$$
\text { (2.12) } \quad d Y=E Y d t+\sum^{1 / 2} d Z
$$


where $E$ and $\sum$ are $(4 \times 4)$ matrices and $Z(t)$ is a vector of standard independent wiener processes. $\sum$ is assumed to be symmetric and positive definite. Without any loss of generality, $\sum^{1 / 2}$ can be taten to be lower triangular with positive diagonal elements. Let $\sigma$ denote the vector of nontrivial parameters in $\sum 1 / 2$.

Switchirg to logarithms and applying Ito's Lemma we can rewrite (2.5) in terms of the $Y(t)$ process as

$(2.1 \Xi) \quad E_{t}\left[d V_{i}(t)-A * d C(t)\right]+\left(g_{i}-A^{*} g_{c}+1 n \delta\right) d t$ $+1 / 2 * A^{2} * \operatorname{Var}(d C)-A * \operatorname{Cov}\left(d C, d V_{i}\right)+1 / 2 * \operatorname{Var}\left(d V_{i}\right)=0$. If this is to hold at all points in time in m.s., then

$$
\text { (2.14) } \quad E_{t}\left[d V_{i}(t)-A * d C(t)\right]=0 \text {. }
$$

The reason is that, according to our assumptions, the remaining terms in the expression are not functions of information. Since the model is homogeneous, the only way this sum can be constant is if it is zero.

Therefore (2.5) imposes the following restrictions on our model :

$$
\text { (2. 15a) } \quad J_{i} B=0
$$$$
\text { (2.15b) } \quad g_{i}-A * g_{c}+1 / 2 * J_{i} \sum_{j}+1 n \delta=0 \quad i=1,2, \Xi
$$

where $J_{i}=\left(-A e_{i}^{\prime}\right)$ and $E_{i} \in F^{\bar{S}}$ is the vector with urity in component $i$ and zero elsewhere.

Suppose that the process $Y(t)$ is sampled at regular intervals. It is straightforward (see Bergstrom[1984]) to show that the point sampled process has the representation

$$
\text { (2.16) } \quad Y(t)=\sigma Y(t-1)+u(t)
$$


where $\sigma=e^{B}$, the matrix exponential of $E$, and $u(t)$ is the random variable $f_{t-1}^{t} e^{E(t-s)} \sum^{1 / 2} d z(s)$. Let $\nabla(t)$ denote the time average of the $Y(t)$ process, i.e. $\nabla(t)=f_{t-1}^{t} Y(s) d s$. Upon integrating both sides of (2.16) we obtain

(2.17) $\quad \nabla(t)=\sigma P(t-1)+\bar{u}(t)$

where $\bar{u}(t)$ is the random variable $f_{t-1}^{t} f_{\tau-1}^{\tau} e^{B(\tau-s)} d Z(s) d \tau$.

Let $f$ and $g$ denote two "smooth" real-valued functions and z(s) a univariate wiener process. Using the definition of the Ito integral, the following two results can be established:

(2.18) $\quad \int_{0}^{T} f(t)\left[\int_{0}^{t} g(s) d z(s)\right] d t=f_{0}^{T}\left[f_{s}^{T} f(t) d t\right] g(s) d z(s)$

(2.19) $E\left[f_{t}^{t} \frac{2}{1} f(s) d z(s)\right]\left[f_{t}^{t} \underline{4} g(\tau) d z(\tau)\right]=f_{M} f(s) g(s) d s$ where $M \equiv\left[t_{1}, t_{2}\right] \Omega\left[t_{3}, t_{4}\right]$

and where the equality is understood in the mean square sense. Applying (2. 1B) element by element and other standard properties of the Ito integral allow us to write

$(2.20) \bar{u}(t)=f_{t-2}^{t-1} \int_{5}^{t-1} e^{B(\tau-s)} d \tau d Z(s)+f_{t-1}^{t} f_{5}^{t} e^{E(\tau-s)} d \tau d Z(s)$. Define $\Omega_{\tau} \equiv E \bar{u}(t) \bar{u}(t-\tau)$ and $F(r, w) \equiv e^{E r} \sum e^{B} w$. Applying (2.19) and standard change of variable rules, we obtain

$$
\begin{array}{ll}
\text { (2.21a) } & \Omega_{0}=f_{0}^{1} f_{5}^{-1} f_{5}^{-1} F(r, w) d r d w d s+f_{0}^{1} f_{0}^{5} F(r, w) d r d w d s \\
(2.21 b) & \Omega_{1}=f_{0}^{1} f_{5}^{1} f_{0}^{5} F(r, w) d r d w d s \\
(2.21 c) & \Omega_{T}=0 \quad \tau=2 .
\end{array}
$$

We conclude that $\nabla(t)$ is a vector AFMA(1,1) process. Finiliips[1978] and Bergstrom[1984] develop similiar results although the latter only considers the case where $B$ is invertible. We can therefore write

(2.22) $P(t)=\Phi 8(t-1)+\epsilon(t)+e E(t-1)$ 
where the inriovations $E(t)$ have mean zero and covariance matrix $S$, and $\theta$ is a matri: with spectral radius not exceeding unity. Define $y(t)=\left(1 n c(t) \text { ln } v_{1}(t) \text { ln } v_{2}(t) \text { ln } v_{3}(t)\right)^{\prime}$, and let $\bar{y}(t)$ be its urit average. Eq (2.22) can be rewritten $a s$

$$
\text { (2.25) } \bar{y}(t)=\gamma_{0}+\gamma_{1} E+\sigma \bar{y}(t-1)+\epsilon(t)+\theta \epsilon(t-1)
$$

where $r_{0}=(I-\phi) t+\phi g, \gamma_{1}=(I-\sigma) g$, and $E=f_{0}^{-1}(t+s) d s$. The restrictions (2.15) are easily shown to imply $J_{i} \gamma_{1}=J_{i}(I-\sigma)$ $=0$. In particular, it also follows that the vector $k$ cannot be identified uniquely. We therefore impose the identification restriction $k_{i}=A * k_{c}$ in our estimation. A tedious argument also shows that

(2.24) $\left(J_{i}-J_{j}\right)(\bar{y}(t)-\bar{y}(t-1))=\left(J_{i}-J_{j}\right) \gamma_{0}+\left(J_{i}-J_{j}\right) \in(t)$

$$
+.268 *\left(J_{i}-J_{j}\right) \in(t-1)
$$

so that the time averaged excess returns on asset $i$ over $j$ follows an MA(1) process with coefficient .268.

To gain further intuition about the possible consequences of $t$ ime averaging suppose $B=d i a g\left(\lambda_{1}, \lambda_{2}, \lambda_{3}, \lambda_{4}\right)$. Then it can be shown that $\Omega_{0}(i, j)=h\left(\lambda_{i}, \lambda_{j}\right) \cdot \sum(i, j)$, where

$$
\begin{aligned}
h\left(\lambda_{i}, \lambda_{j}\right) & =\left(\lambda_{i} \lambda_{j}\right)-1\left[1+e^{\lambda i+\lambda j}-2 *\left(\lambda_{j}+\lambda_{j}\right)-1\left(1-e^{\lambda i+\lambda j}\right)\right. \\
& \left.+\left(1+e^{\lambda_{i}}\right)\left(1-e^{\lambda j}\right) / \lambda_{j}+\left(1+e^{\lambda j}\right)\left(1-e^{\lambda_{i}}\right) / \lambda_{j}\right]
\end{aligned}
$$

and equal to the obvious limits as $\lambda_{i}$ or $\lambda_{j}$ goes to 0 . Our simple example corresponds to the case $h(0,0)=2 / 3$. If the process were stationary around trend, the eigenvalues of E would have negative real parts. Sampling a few values, we see that $h(-.1,-.1)=.60, h(-.5,-.5)=.45, h(-1,-1)=.28$, and $h$ goes 
to zero as $\lambda_{i}$ and $\lambda_{j}$ both go to minus infinity. The bias in the estimate of A Lising time averaged data and (2.5) can therefore be arbitrarily large. 1

\section{DATA ANALYSIS}

\section{Data Description}

The data are fully described in an appendix to this paper which is available from the authors. Here we shall give only a broad description of the data to indicate how they were assembled and to show that they correspond as much as possible to the concepts represented in the model above.

Six separate data sets were prepared, each intended to represent a series of observations on the four-element vector $\bar{y}$. The data sets differ in sample period, sources and assumptions about taxation. Table 2 summarizes the important differences.

Data sets one and two are long historical annual time series beginning in the year 1890. These data sets are based on those used in Grossman and Shiller[1981] and described also in

Shi]ler[1982]. Data sets three through six are quarterly time series. Data sets three and four begin in the second quarter of 1953. Data sets five and six begin in the second quarter of 1947. The use of annual and quarterly time series was dictated

\footnotetext{
$I_{A}$ more relevant comparison might be the ratio of $A$ that would be obtained using time averaged data to that using point sampled data. Although details differ, it is easily shown that this ratio also can be arbitrarily large.
} 
by the existing consumption data. Long time series data or consumption are available orily on an annual basis. Quarterly consumption data are available only for the post-war period. Monthly consumption data are available starting in 1959. We did not use those data here because of some concern as to the accuracy of the monthly data and because of the somewhat shorter sample period that such data would impose.

In all data sets, the first element of $\bar{y}$ is the log of real per capita seasonally-adjusted consumption on nondurables and services. For years beginning with 1929 these data are from the National Income and Froduct Accounts of the United States. Earlier data are the fuznets-kendrick: series. Since the published consumption series are total consumption over the period, the first elemerit of $\bar{y}$ departs somewhat from that hypothesized in the paper: it is the log of the integral rather than the integral of the $10 \mathrm{~g} .2$ Note that we use a physical measure of consumption directly and do not deflate nominal consumption by a price index that is averaged over the year, which would have introduced another departure from the assumptions of our model.

In all data sets the second element of $\bar{y}$ is a measure of the interval averaged $l o g$ cumulated real return on corporate stocks, the third element is a measure of the interval averaged log cumulated real return on short debt and the fourth element

\footnotetext{
ZSome Monte Carlo simulations indicate that the biases introduced by using the log of the average instead of the average of the log are extremely small, at least for our data.
} 
is a measure of the interval averaged log cumulated real return on long-term bonds. The even-numbered data sets are based on after-tax returns. In constructing these series, the (after-tax in even-numbered cases) nominal returns were first computed on a monthly basis. At that point, a choice had to be made whether to use the consumption deflator to convert nominal returns to real returris or to use one of the monthly price indices for this purpose. The consumption deflator has the advantage that it corresponds to the measure of consumption that is supposed to enter the lutility function. The monthly price indices have the advantage that we can use them to produce a monthly real series, so that our interval average will correspond more clasely to the integral of the log of the real portfolio value as represented in our model. It was decided to use the consumption deflator for data sets one through four and the monthly consumer price index for data sets five and six. Thus, for example, the second through fourth elements of the $\bar{y}$ vector in data set two were constructed by first producing monthly series representing the cumulated after-tax nominal returns of the assets. Each series represented the nominal value of the portfolio of an individual who reinvests all after-tax income from the asset in the same asset. 3 The average for the year of the 109 of the monthly portfolio values was used to construct an annual series. Finally, the log of the consumption deflator was subtracted

3 Let $\left(1+r_{i m}\right)$ denote the monithly after-tax riominal return on asset $i$, and $l$ et $v_{i}^{n}$ denote the cumulated after-tax return in month $L$. We set $v_{i L}^{n} \stackrel{=}{=}\left(1+r_{i 1}\right)\left(1+r_{i 2}\right) \cdots\left(1+r_{i L}\right)$. 
from each series to convert to a real series. With data set five, the first step in the construction of the second through fourth elements of $\bar{y}$ was essentially the same. We first produced a monthly series of cumulated returns of the assets. However, in data set five, this monthly series was subsequently deflated by dividing by the consumer price index, and a quarterly series was produced as the average for the three months of the quarter of the log of this monthly real series.

With data sets five and six another adjustment was also made before the average log cumulated real portfolio value was entered into the vector $\bar{y}$. In constructing the series, there was great concern that the data be aligned properly. The Ibbotsori-sinquefield returns data for each month are measured from the end of the preceding month to the end of the current month. This provides four point sampled observations on the log cumulated real portfolio for each quarter. These were connected Ey straight lines and the integral under the straight 1 ine interpolation was used to estimate the corresponding component of $\bar{y}$.

For data sets one and two, the return on corporate stocks is computed from the Standard and Foor's Composite Stock Frice Index and associated dividend series. The return on short-term debt is computed from the prime commercial paper rate and the return on long-term debt is computed using the Macaulay railroad bond yield data for the first part of the sample and the Moody Aaa bond yield average for the years after 1936. 
For data sets three and four, all return data come from series on the CITIEASE data library. Stock: returne are again computed using the monthly Standard and Foor's Composite Stock: Frice Index, while the return on short debt is taken from the return on three-month treasury bills and the return on long debt is based on yields of twenty-year treasury notes.

For data sets five and six, return data come from Ibbotson and Sinquefieid[1982]. The stock return series is their series comimor stocks, total returns: the short debt series is their series U.S. Treasury bills, total returns; the long debt return series is their series long-term corporate bonds, total returns. For after-tax series, the assumed marginal income tax rate for 1918 to 1980 was that implicit in the spread between municipal and corporate bond yields. Before 1918 , the marginal income tax rate was set to zero. Since the Ibbotson and Sinquefield data do not allow a decomposition of returns into capital gains and income comporients, it was assumed for data set six that all returns were taxed each month as income. For data sets two and four, however, capital gains were assumed taked each month at a long-term capital gains rate. For the years 1946 to 1978 , the effective rate on long-term capital gains was orie-half the marginal income tax rate. For earlier years, the effective rate on long-term capital gains was computed from the marginal income tax rate using tax rate data in seltzer[1951]. 


\section{Freliminaries}

Eefore considering formal estimation and testing, it is useful to review some of the broad features of the si\% data sets which our model must explain. Some descriptive statistics are provided in Table $\mathbf{S}$.

For all six date sets, we observe that stock portfolios gave the highest average real return, approximately 6\% p.a. on a pre-tax basis or $4 \%$ after-tax. Short-term paper yields averaged about $2 \%$ p.a. on a pre-ta: basis over our longest historical sample, but the average yield fell to about zero in the postwar period. After-tax real returns to holding short-term paper have beer, slightly riegative. Long-term bonds, by contrast, have averaged essentially a zero real return over the last century, on both a pre- and after-tax basis. During the post-war period, however, pre-tax returns have been slightly negative. On an after-tax basis, boridholders have seen the real value of their portfolios shrint: by over $2 \%$ p.a.

fccording to the consumption based asset pricing model, these persistent differences in average yields must be accounted for by the insurance provided by the different portfolios against events which impinge adversely on consumption. Useful evidence about this hypothesis is obtained by looking at the covariance structure of measured portfolio yields and changes in consumption. Some caution is recessary since the model's predictions pertain to the covariance structure of the instantaneous returns and 
15

our data are constructed from differences of unit averaged values.


the sign and order of magnitude of the instantaneous covariance matrix.

Several empirical regularities emerge. As measured by the variance, the change in consumption is the smoothest series, followed closely by the yield on short-term paper. Long-term bond yields have been fairly stable over our longest sample, whereas the variance of returns to holding a portfolio of stoct:s has been several orders of magnitude 1 arger. In the post-war period, real returns to holding long-term bonds have been much more volatile with a variance almost as large as the return to holding common stocks.

Of more interest are the covariance properties. According to our model, it is not the variance but the covariance with consumption that is the relevant measure of a portfolio's rist: We find, uniformly across the si: data sets, that stock yields have the largest covariance with changes in consumption, followed by short-term paper yields and then yields on long-term bonds. Qualitatively, this is exactly what the model requires given the ordering of the average yields. It indicates that the basic idea that insurance against adverse movements in consumption can account for observed yield differentials has some empirical promise.

Evidence of potential difficulties is provided by the autocovariance structure of excess returns on bonds and stocks 
over short-term paper. Given our assumptions about the protabilistic structure of corsumption and portfolio values and the form of preferences, we expect the point sampled difference in yields between ary two portfolios to te serially uncorrelated. As equation (2.24) shows, the time averaged difference in yields should have an MA(1) comporient with coefficient about . 268 . This particular prediction is independent of the mean or covariance of returne or the degree of relative risl aversion.

Table 3 shows that it is important to take into account the corisequences of time averaging. The Eox-Ljung statistics clearly indicate that the excess yields that are constructed from our data are not white noise. The adjusted excess returns referred to in Table 3 are filtered to remove the time dependence that is induced by unit averaging. Judging from the Eox-Ljung statistics, the adjusted excess returns are indeed less serially correlated. Nonetheless, the autocorrelations of the adjusted excess returns to stoclss remain statistical1y significant from zero in four of the six data sets.

\section{Some Econometric Issues}

It is demonstrated above that the vector of time averaged observations has a representation of the form $(3.1) \quad \bar{y}(t)=\gamma_{0}(\alpha)+\gamma_{1}(\alpha) E+\sigma(\alpha) \bar{y}(t-1)+E(t)+\theta(\alpha) \in(t-1)$ where the disturbances $\epsilon(t)$ are distributed independently and identically as MUN $(0, S(\alpha)$ ). In our application, we can 
set $\alpha^{\prime} \equiv\left(k_{c}, g_{c}, A, \delta, B_{1}, \sigma\right)$, where $B_{1}$. denotes the first row of the 8 matrix.

Linear Gaussian processes have been studied extensively by econometricians arid statisticians. Nonetheless, there are several features of our model which put it outside of the standard assumptions in the literature used to prove laws of large numbers or central limit theorems. First, the model contairis a time trend so that sample autocovariances of the exogenous variables, i.e. $T^{-1 \sum x_{t} x_{t-j}^{\prime}}$ where $x_{t}^{\prime}=(1 \mathrm{t})$, do rot converge to well defined limits. Secondly, the model imposes restrictions not only across the autoregressive and moving average matrices, but across these and the contemporaneous covariance matrix as. well. Finally, our model imposes the restriction that $B$ be of rant: one, so that o( $\alpha)$ will have three eigenvalues on the unit circle. To our knowledge, there are no 1 aws of 1 arge numbers or central limit theorems that cover all three of these features. Application of the standard large sample procedures to estimate and test our model must be considered teritative.

A] though al the features of our model have not been treated together in the literature, we can use available results to form a reasoriable guess about the samplirig properties of the approximate (conditional) maximum likelihood estimator described below. For example, it appears that a law of large numbers which would allow for all three of the features noted above would be a modest extension of the literature. Hannan et al.[1980] provide a law of large numbers for vector ARMAX models al lowing 
for very general restrictions and, in particular, dependence across the covariance matrix of innovations and the other parameters of the model. Their assumptions about the error process are cleatly satisfied by our model, but they rule out time trends as regressors and require all roots of the autoregressive polynomial to be outside the unit circle. In the absence of complicated restrictions or unit roots, the assumption that sample covariances converge to well defined limits can be replaced by the weat:er Grenander conditions (see Hannan[1971]) which do allow for time trends as regressors. Similarly, in the absence of time trends and other restrictions, strong laws of large numbers can be established even if the autoregressive process is explosive. Individually, therefore, each of the three features of the model highilighted above is rot an impediment to establishing a law of large numbers.

It is well linowr that unrestricted estimates of $\sigma$ will not be asymptotically normal if there are unit roots in the autoregressive polynomial. A case for a central limit theorem can be made only if the estimation procedure exploits the prior knowledge of the structure of $d$. Dur restrictions imply that $\bar{y}_{t}$ is a co-integrated process (see Granger-Engle[1982]). These processes have had a long history in applied empirical research under the name of "error-correction" models. However, only recently has there been any serious investigation of the sampling properties of the MLE or its approximants. Available theorems do not allow for a time trend or moving average terms but these 
complications do not appear to present any conceptual difficulties. The main result is that the integratirig factor ${ }^{4}$ is estimated consistently by ML with a sampling error that is $o_{p}\left(T^{-1 / 2}\right)$. The ML estimators for the remaining parameters are consistent and asymptotically normal with a covariarice matrix that is estimated consistently by the usual formula. In our model, the integrating factor is just $\mathrm{B}_{1}$. , appropriately scaled. Since we are riever concerned with testing restrictions on the components of $E_{1}$., the rapid convergence of the estimated integrating factor does not appear to present a problem.

We will proceed formally as if the standard large sample procedures for inference are valid under the maintained hypothesis that $B$ is of rank one. As the preceding discussion makes clear, however, some scepticism is in order.

\section{Estimation Strategy}

Several strategies for the estimation of models with MA errors have been proposed. 5 In the time domain, it is natural to consider the maximum likelihood estimator, or one of its various approwimants.

Fut $e(1) \equiv 0$ and for ariy admissable or define $e(t)$ recursively according to

\footnotetext{
${ }^{4} \mathrm{~A}$ nonstochastic vector $c$ such that $c \cdot \bar{y}_{t}$ is stationary is called an integrating factor. In our application, it is any normalized basis vector for the row space of $I-o$.

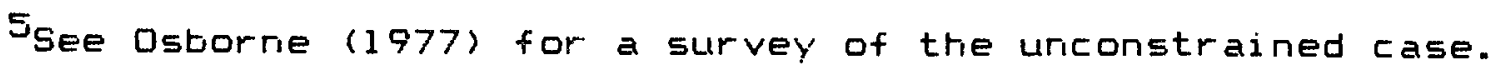


$(3.2)$

$e(t)=\bar{y}(t)-\gamma_{0}(\alpha)-\gamma_{1} E-\phi(\alpha) \bar{y}(t-1)-\theta(\alpha) e(t-1)$.

Following Wilson ( 1973 ), we choose as our estimator $\alpha_{a}$,

the admissable vector $\alpha$ which maximizes the approximate

(conditional) log likelihood function

$$
\begin{gathered}
L_{a}(\alpha) \equiv-\frac{(T-1)}{2} \ln |S(\alpha)|-\frac{1}{2} \operatorname{tr} S^{-1}(\alpha) M \\
\text { where } M \equiv \sum_{t=2}^{T} \text { e(t)e(t)'. }
\end{gathered}
$$

since 0 has unit roots, we have little choice but to condition on the first observation $\bar{y}(1)$. The assumption that $e(1)=0$, by contrast, is made solely out of convenience. If $B=0$, the spectral radius of $\theta$ is about. 268, so the sampling distribution of $\hat{\alpha}_{a}$ will rot be very sensitive to this assumption about the initial innovation. Futting $e(l)=0$ does simplify the computations somewhat. In particular, analytic derivatives can be easily and quickly computed lusing the method of adjoints and a etraightforward application of the chain rule.

Several features of $L_{a}(\alpha)$ mate the evaluation of $\hat{\alpha}_{a}$ challenging. As with any model with MA errors, it is not possible to reduce the data through sufficient statistics and we have to deal with a likelihood function that is not guaranteed to be globally concave. Dur model poses several difficulties in addition to these standard ones. For example, it is not possible to concentrate out the covariance matrix, since 5 is functionally related to the regression parameters of $(3.1)$. Also, some effort is required to evaluate $(\sigma(\alpha), S(\alpha), \theta(\alpha))$. Details are provided 
in Melino[1985], so we will give only a brief overview here. Define the matrices

$$
(\Xi .4) \quad C \equiv\left[\begin{array}{rrrr}
-E & I & 0 & 0 \\
0 & -E & \sum & 0 \\
0 & 0 & 0 & I \\
0 & 0 & 0 & B
\end{array}\right] \quad e^{C} \equiv\left[\begin{array}{llll}
F_{1} & G_{1} & H_{1} & V_{1} \\
0 & F_{2} & G_{2} & H_{2} \\
0 & 0 & F_{3} & G_{2} \\
0 & 0 & 0 & F_{4}
\end{array}\right] .
$$

Fut $\tilde{C}=-C$ and denote the blocks of $e^{\tilde{C}}$ by $\tilde{F}_{1}, \tilde{G}_{1}$ etc. It can be shown that

$$
\begin{aligned}
& \text { (S.5) } \Omega_{0}=F_{4} F_{1}+\digamma_{1} F_{4}-\tilde{F}_{1} \tilde{F}_{1}-\tilde{F}_{1} \tilde{F}_{1} \\
& \text { (S.6) } \Omega_{1}=\tilde{H}_{1} \sigma_{3}+\tilde{F}_{1} \tilde{F}_{1}+\tilde{F}_{1} \tilde{F}_{1} .
\end{aligned}
$$

It is also useful to note that $\sigma=F_{4}$. Although the expressions appear to be unappetizing, they are straightforward to implement given an algorithm for computing the matrix exponential. We used a routine based on a diagonal Fade approximation that has very nice numerical properties.6

Solving for $(S, \theta)$ given $\left(\Omega_{0}, \Omega_{1}\right)$ turned out to be much easier than conjectured by Bergstram[1984]. Wilson[1972] provides a gerieral algorithm for factoring the autocovariance function of a multivariate MA process. We adapted his suggestion to our special case and applied Newton's method to find the matrix $\theta$ with spectral radius no greater than unity which is a root of the polynomial

$$
\text { (3.7) } \quad \Omega_{1}-\theta \Omega_{0}+\theta \Omega_{1} \theta=0 \text {. }
$$

Given an initial guess, $\theta(0)$, this leads to the iterative scheme

\footnotetext{
GWe would lit:e to thank: Dr. Fi.C. Ward of the Union Carbide Laboratory
} in Dak: Fidge for lindly providing us with this code. 
(..8) $\quad \theta(n+1)\left[\Omega_{0}-\Omega_{1} \theta^{\prime}(n)\right]-\theta(n) \Omega_{1} \theta^{\prime}(n+1)=\Omega_{1}-\theta(n) \Omega_{1} \theta^{\prime}(n)$.

This scheme exhibits quadratic convergence and turns out to be quite fast. On average, less than three iterations were required to find $\theta$ given $\left(\Omega_{0}, \Omega_{1}\right)$. In fact, we found that this scheme rarely required more than 5 iterations. Given $\theta$, it is straightforward to solve for 5 using $S=\Omega_{0}-\Omega_{1} \theta^{\prime}$.

Evaluation of $L_{a}(\alpha)$ and its analytic derivatives is fairly quict and easy. The main difficulty in computing $\alpha_{a}$ turned out to be the extraordinary large number of iterations required to refine its location.

\section{Farameter Estimates}

Tatle 4 presents the estimated parameters of the constrained model for each of the the six data sets. 7 The estimates obtained using before- and our constructed after-ta\% yields are remarliably similiar, but there are considerable differences in the estimates across the three different sample periods.

Consider first the estimates of $\Sigma$, the covariance matrix of the instantaneous innovations. Once again, correlations are displayed above the diagonal, and the lower triangular elements are covariances. The estimates of $\sum$ from the quarterly data sets are all similiar. However, there are some sharp contrasts

\footnotetext{
${ }^{7}$ Estimates were obtained usirag the GQOFT3 package provided by Frofessor Quandt of Frinceton University. Various algorithms were required to refine the location of $\alpha_{a}$. The reported standard errors, however, are al ways calculated by inverting the matrix of second derivatives evaluated at the optimum. The Hessian was computed using symmetrical numerical differences of analytic first derivatives.
} 
with the estimates from the annual samples which cast doubt on our assumption that $\sum$ has been constant over time. Consumption innovations appear to have had a much smaller variance in the post-war period, as have had the innovations to the value of short-term paper. By contrast, the innovations to stock market values have been slightly smoother, and those for long-term bonds are roughly comparable. The covariances of the innovations to portfolio values with consumption have the same ranting in all six data sets, but they are much smaller in the post-war period.

All six data sets yield small estimates of $B_{1}$, the first row of the $\mathrm{E}$ matrix. This indicates that the change in consumption has only a very small predictable component, aside from trend. The trend in consumption is estimated to be about $3 \%$ p.a. Lsing the two long historical samples, about $2.5 \%$ using data sets three and four, and about $1.6 \%$ p.a. using data sets five and six. The corresponding point estimates for $\varepsilon$ indicate, respectively, a substantial preference for present consumption, a substaritial preference for future consumption, and indifference. These apparent differences can't be taken too seriously since the estimated standard errors indicate substanial uricertainty.

The differences in the estimated parameters of relative rist aversion are extremely interesting. Using our two longest historical samples, we obtain estimates of A of just over 20 . This is too large to be plausible. Nonetheless, as ariticipated, 
accounting for unit averagirg of consumption results in a substantial reduction. 8

Data sets five arid six produce a very plausible estimate of A of just over 2. Ey contrast, data sets three and four produce an Estimate of A over 150! The difference of the parameter estimates obtained using these very similiar post-war quarterly data sets is very large, and some clarification is in order. The estimates of $A$ presented in Table 1 are derived from restrictions which relate the unconditional means to the covariances of consumption changes and portfolio returns. However, the model provides us with further sources of information about A. Equation (2.14) telis us that the predictable change in the value of any portfolio is equal to a multiple of the predictable change in consumption, up to a constant. Since the multiple is just the parameter of relative rist aversion, this gives us another estimate of $A$ based on the conditional information in the sample. The maximum litelihood estimator is usefully viewed as suitably pooling the disparate estimates based on conditiorial arid unconditional information.

It turned out that the predictable change in consumption around its mean using the lagged information in data sets $1-4$ was essentially zero. As a result, the maximum likelihood estimate of A closely reflects the estimates in Table 1 adjusted for unit averaging. In data sets 5 and 6 , however, the predictable

B For data set 1 , we also estimated the model as if the data was actually point sampled. We obtained an estimate $\hat{A}=27.24$, with a standard error of about 11.2 . 
charge in consumption about its mean, while still small, was large enough to provide a fairly accurate estimate of $A$. The maximum litelihood estimate reported for these data sets reflects the conditional information in the sample. 9

Our model imposes restrictions on the time averaged representation of $\bar{y}$. In turn, the time averaged representation imposes additional structure on the parameters of the $\operatorname{AFM} A(1,1)$ representation. Table 5 contains the log libelihoods, denoted $L_{1}, L_{2}$, and $L_{3}$ respectively, for the fully restricted time averaged estimates (Model 1 ), the unconstrained time averaged estimates (Model 2), and the uncoristrained AFiMA $(1,1)$ estimates (Model $\overrightarrow{;}$ ). For the reasons discussed above, Models 2 and $\Xi$ were estimated under the maintained hypothesis that $\mathrm{B}$ is of rank one and 6 is the sum of the identity and a rant one matrix. For completeness, the log litelihoods for the totally unconstrained time averaged and AFMA $(1,1)$ models, Ĺ் and L\$் respectively, are also reported. The tests of the overidentifying restrictions imposed by the model are rejected with very high confidence when compared against either Model 2 or Model 3 . Curiously, data sets three and four which produced the least plausible parameter estimates,

9Imposing only the restrictions implied ty (2.14), we obtained for the six data sets:

$$
\begin{aligned}
& \ddot{A}=663.54 \cdot 949.86 \quad 439.67 \quad 985.73 \quad 2.07 \quad 2.60 \\
& \text { (5.e.) (370.56) (370.56) (*) (*) (0.92) (1.21) }
\end{aligned}
$$

Because the Hessian was singular, we are uriable to provide standard errors for the estimates from data sets three and four. Hansen-singleton[1983] also report a very sharp differerice in the estimate of A depending upon whether or not conditioning iriformation is used. (See their Table 5) 
provide the weat:est evidence against the overidentifying restrictions. Finally, a comparison of $L_{Z}$ and $L_{3}$ indicates that there is some difficulty in accounting for the autocovariances of $\bar{y}$ by time averaging a first order process.

Why is the model rejected?

There are strong a priori reasons for linking corisumption and portfolio choices. Moreover, the sample means and covariances of portfolio yields and changes in consumption lend qualitative support to the notion of assets being priced in accordance with the insurance they afford against adverse movements in consumption. Yet the various goodness of fit tests reported above as well as the implausibly high estimates of relative rist: aversion from data sets one through four appear to constitute an overwhelming rejection of the model. What should we conclude?

A response that cannot be dismissed is that the assumed distritution of the goodness of fit tests is simply misleading. As we noted above, we cannot rely on the standard central limit theorems to establish the asymptotic distribution. Moreover, even if the large sample results obtain, as we conjecture, there is no guarantee that the asymptotic distribution provides a close approximation for samples of the size we have examined. Unfortunately, establishing the small sample distribution either analytically or by Monte Carlo methods is infeasible. We choose 
to take the evidence against the model seriously and to focus attention on the specific sources of predictive failure.

One is naturally led to examine more closely the various auxiliary assumptions that are teing tested jointly alongeide the hypothesis that agents behave as described by (2. 3 ). The two most obvious are the stochastic process assumed to describe the evolution of consumption and portfolio values and the specific form of preferences. We will concentrate on the former.

The stochastic differential equation (2.12) imposes many overidentifying restrictions. Dne of them is that the time averaged vector has an AFMA $(p, q)$ representation with $p=q=1$. To test this, the autocorrelations of the prediction errors from Model J were calculated. Box-Ljung tests did not indicate any need for considering a higher order process.

Although the evidence suggests that an AFMA(1,1) representation for $\bar{y}$ is a reasonable approximation, there are froblems in accepting the restrictions that time averaging a first-order process imposes on this representation. Fhillips(1978) shows that if Bzo then $\sigma \approx I+B$ and $\theta \approx 268\left(I+\left(E-\sum E \cdot \Sigma^{-1}\right) / 4\right)$. Dur unconstrained $A F M A(1,1)$ estimates of $\sigma$ suggest that $B$ is indeed small. There is little difficulty in accepting the restrictions which a small B matrix and time averaging impose on 6 . However, this combination imposes a great deal of structure on $\Theta$ which is at odds with the data. For all six data sets, we found that both the constrained and unconstrained time averaged models produced estimates of $9 \approx 268 I$. The unconstrained AFMA $(1,1)$ estimates of $\Theta$ differed from . 2681 
ir several respects. The most roticeable discrepancy was that the unrestricted estimate of the row of the moving average matrix pertaining to the consumpton equation was essentially aero, in all six data sets. In fact, in data sets $1-2$ and $5-6$, the MA coefficient for the innovation in consumption was more than two standard deviations below.268. Failure to explain the MA component of consumption in and of itself would lead to rejection of the model at the $5 \%$ level for these data sets.

Qrie possible explanation for the apparent absence of a moving average component in the consumption equation is measurement error. Suppose the unit average of consumption is measured with an error that is serially uncorrelated and independent of the true consumption process. If the flow of consumption is truly a random walk, the measured consumption series will be an AFMA (1,1) process but with an MA coefficient less than .268. If orie half of the variance of the change in measured consumptiori is due to measurement error, the MA coefficient would be predicted to fall to just .127.

As pointed out earlier, our model predicts that the excess returns of stocks and bonds over the yield on short-term paper should be unipredictable. The time averaged excess returns should therefore have an MA(1) structure with a coefficient of about .268. These overidentifying predictions can be tested regardless of the quality of the consumption data by simply regressing the adjusted excess returns on various information sets. Moreover, there is no problem in justifyirig the standard procedures to 
test these orthogonality restrictions. The results are reported in Tatle 6. The litelihood ratio test statistic, $\lambda$, and the $\mathrm{F}^{2}$ for each of the individual regressions is also reported. The individual. $F^{2}$ are remartiably high and the orthogonality restrictions are rejected with very high confidence. Since yield data that are point sampled are readily available, we also tested these restrictions using the monthly point sampled yields corresporiding to data sets $1-6$. Eecause a monthily price inde: was not available for our longest samples, we used the 109 cumulated nominal returns, vit, in the information set. These results are reported in the lower half of Table 6 . Although the individual $\mathrm{K}^{2}$ are much lower, as we would expect, the rejection of the orthogonality restrictions is even more pronounced.

These results are very similiar to those reported in Harisen and Singleton[1983].

Orie explanation for this predictive failure is simply that the covariance matrix of the instantaneous innovatioris is not constant but is state dependent. This seems extremely plausible and could also account for the noted differences in the estimates of $\sum$ from different sample periods. However, tatiing accourit of state dependent variances would make estimation and testing of the model practically impossible. Eecause our model imposes restrictions across the drift and diffusion parameters, maling the latter state dependent would force us to abandon the linear constant coefficient model of the drift as well. We would be led to the more general stochastic process that solves 
$(3.9) \quad d y=B(t, y) d t+\sum 1 / Z(t, y) d z$.

The restrictions across the drift and diffusion effectively

rule out any of the convenient functional forms for E(*) and $\sum(\cdot)$, and the solution of the lit:elihood for even the point sampled process is difficult to implement. Computing the likelihood function for the unit averaged process that solves (3.9) seems unimaginable, with current technology.

\section{Conclusions}

The notion of insurance against events which impinge unfavourably on consumption choices can be used to rationalize, at least qualitatively, the systematic differences in average yields afforded by portfolios of stocks, bonds, and short-term paper. The sample means and covariances of portfolio returns and per capita consumption growth indicate that the quantitative differences in average yields can be rationalized only by implausibly high aversion to rist. Taking account of the fact that measured consumption is unit averaged substantially reduces the degree of relative risk aversion required to rationalize the data. Nonetheless, there remains considerable evidence that casts doubt on this view of the world. In particular, it is difficult to reconcile the importance of unit averaging of the consumption flow with the fact that the measured logarithm of detrended real per capita consumption has essentially no moving average component. Also, although the model allows the average 
return on different portfolios to diverge due to different insurance characteristics, the particular specification that we examined requires that expected excess returns should be time invariant. This orthogonality property is forcefully rejected by the data. Addressing these particular predictive failures while taking account of unit averaging constitutes a formidable challenge for future research. 







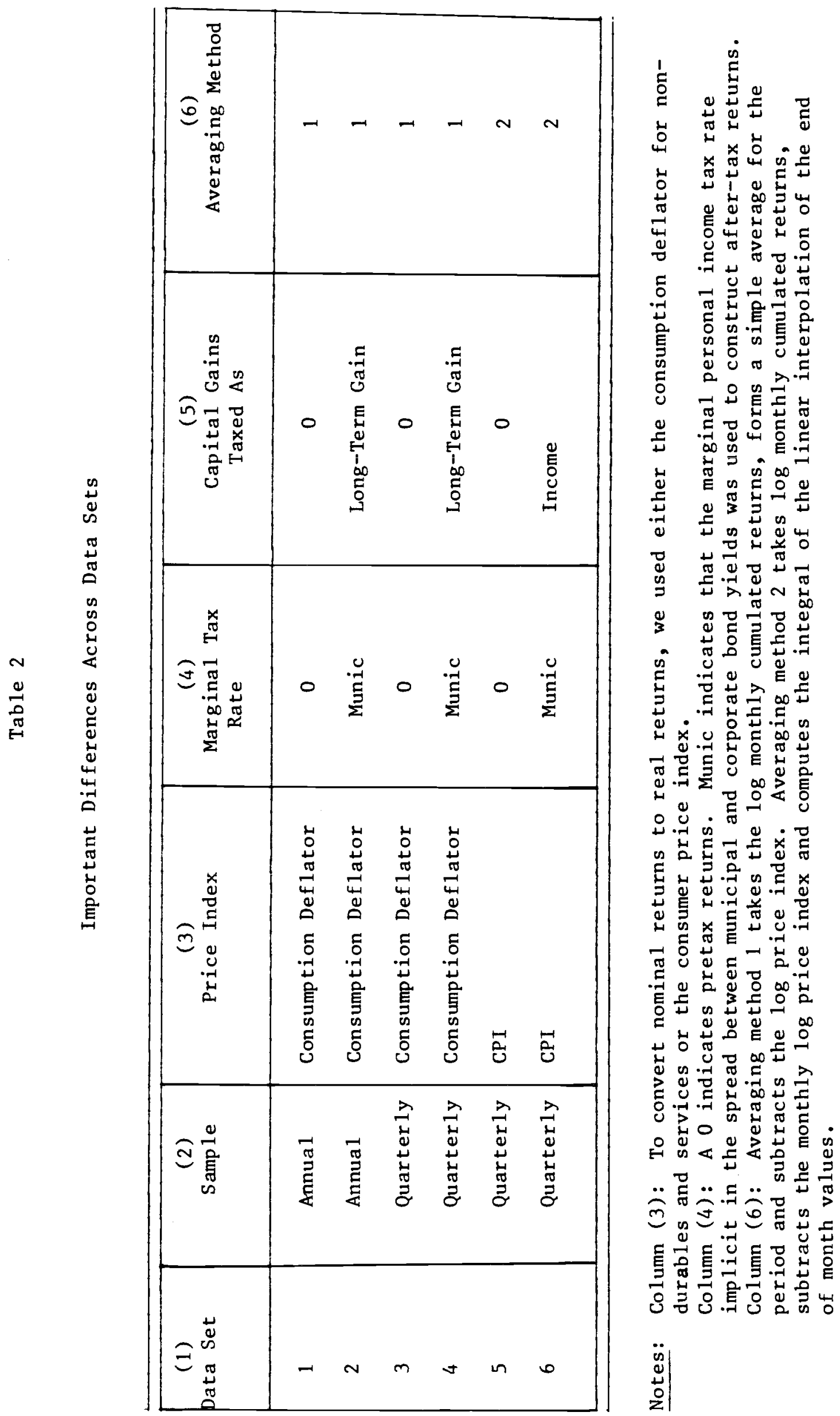




\begin{tabular}{|c|c|c|c|c|}
\hline & 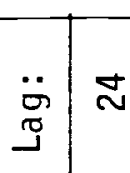 & 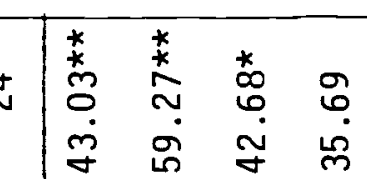 &  & 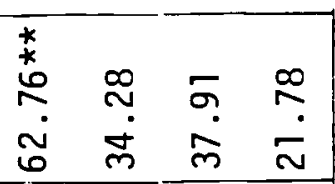 \\
\hline & 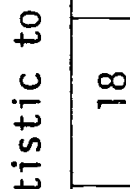 & 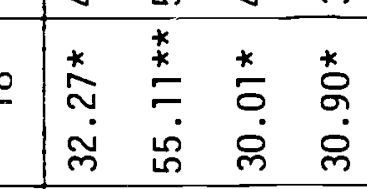 & 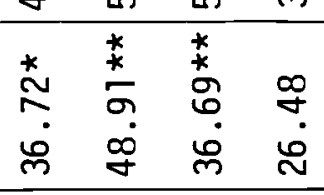 & 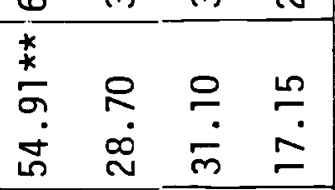 \\
\hline & 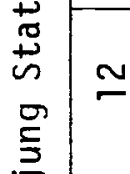 & 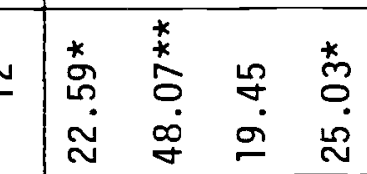 & 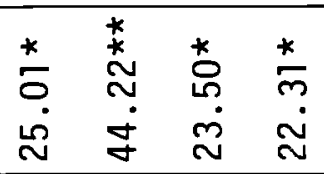 & 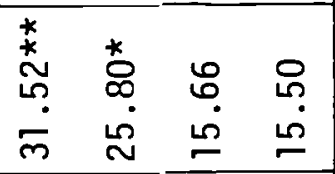 \\
\hline & 宵 & 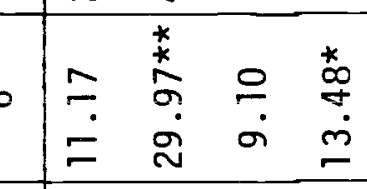 &  & 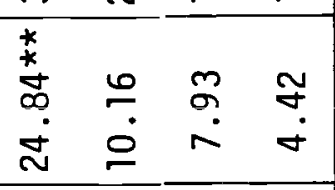 \\
\hline & 胥 & 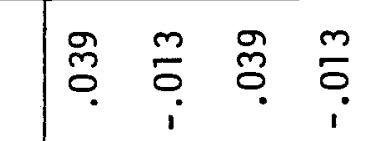 & $\tilde{m}_{0}^{\infty} \bar{\sigma}_{i} \tilde{O}_{0}^{\infty} \bar{\sigma}_{i}$ & 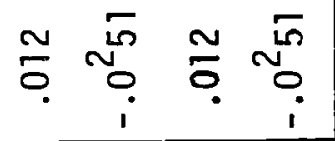 \\
\hline & 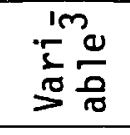 & $\begin{array}{l}x \\
x \\
+ \\
+\end{array}$ & $\begin{array}{l}x_{0}^{\infty} \\
+ \\
+ \\
\end{array}$ & \\
\hline  & $\underset{ِ}{ }$ & 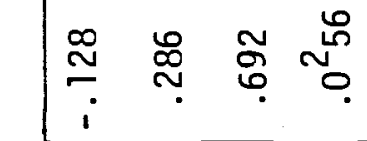 & 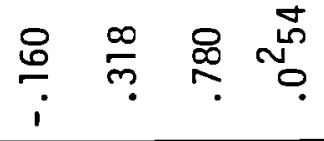 & 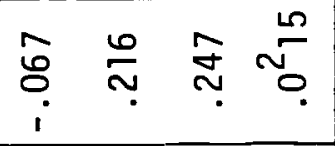 \\
\hline Ф̆ & $\frac{\pi}{2}$ & 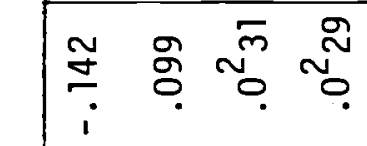 & 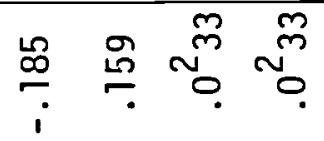 & ڤ్ \\
\hline & 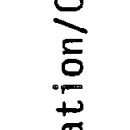 & ه్ & অٓ &  \\
\hline & 离 & 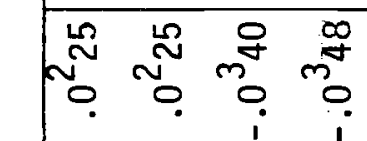 & 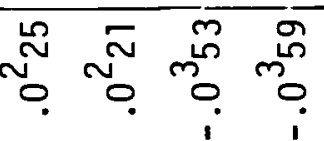 & \\
\hline & $\frac{j}{2}$ & 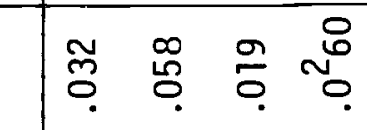 & 范 & 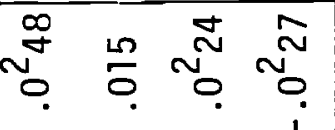 \\
\hline & 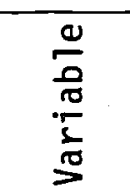 & 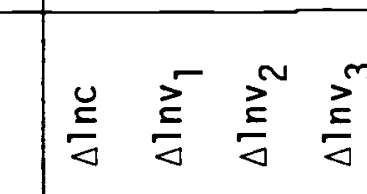 & 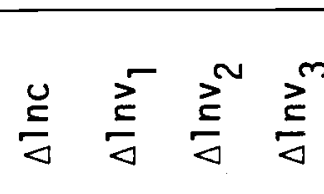 & 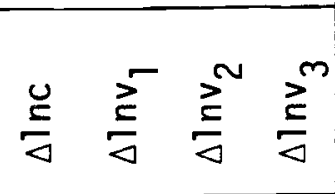 \\
\hline & & 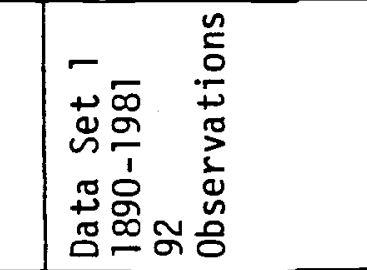 & 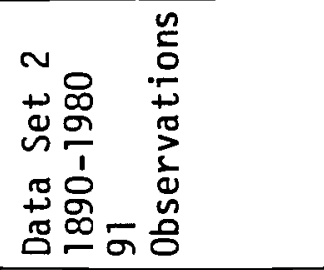 & 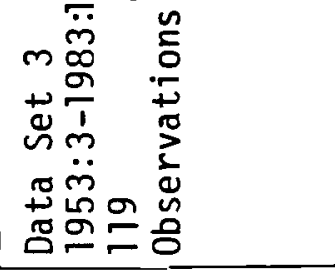 \\
\hline
\end{tabular}




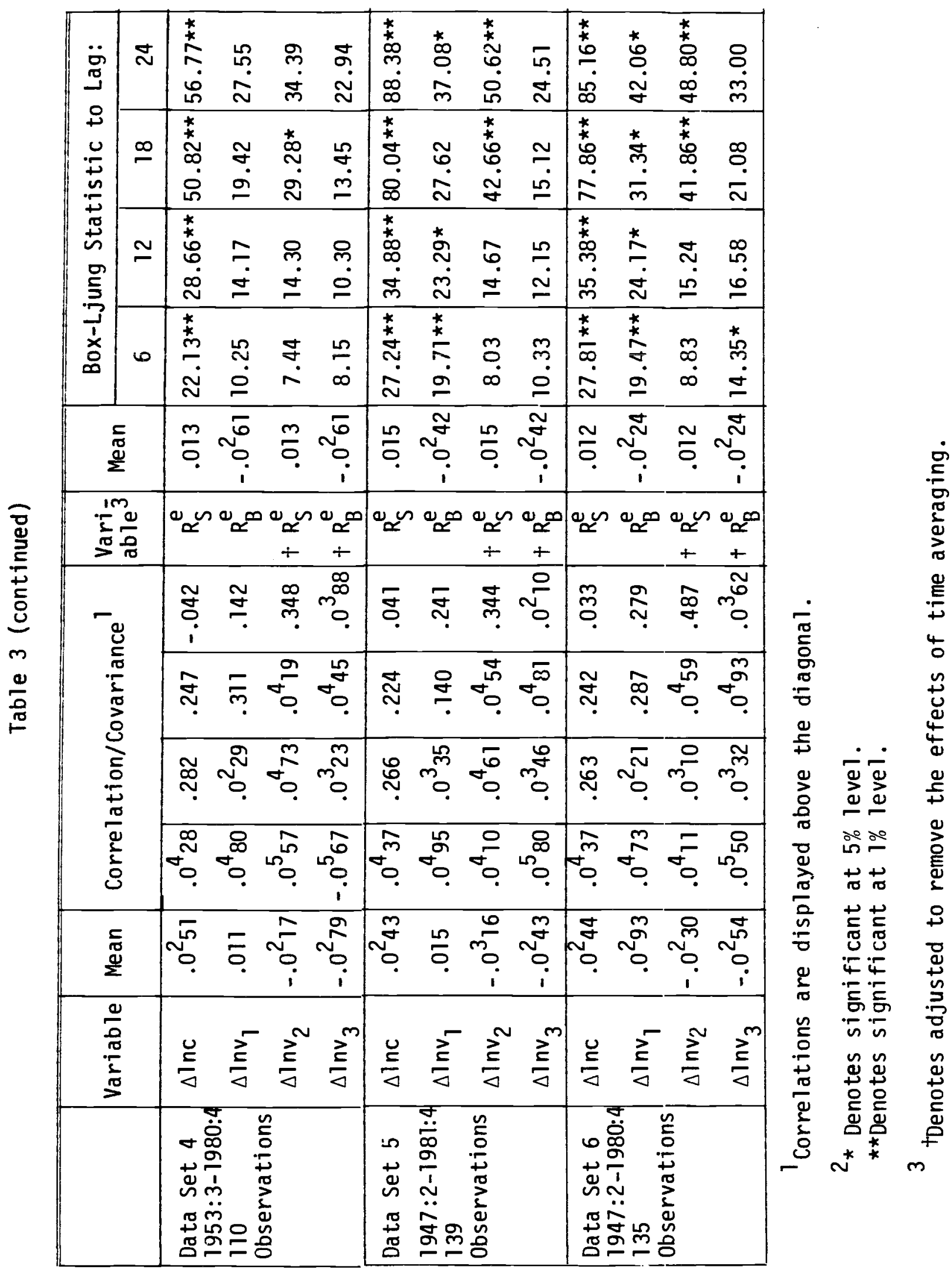




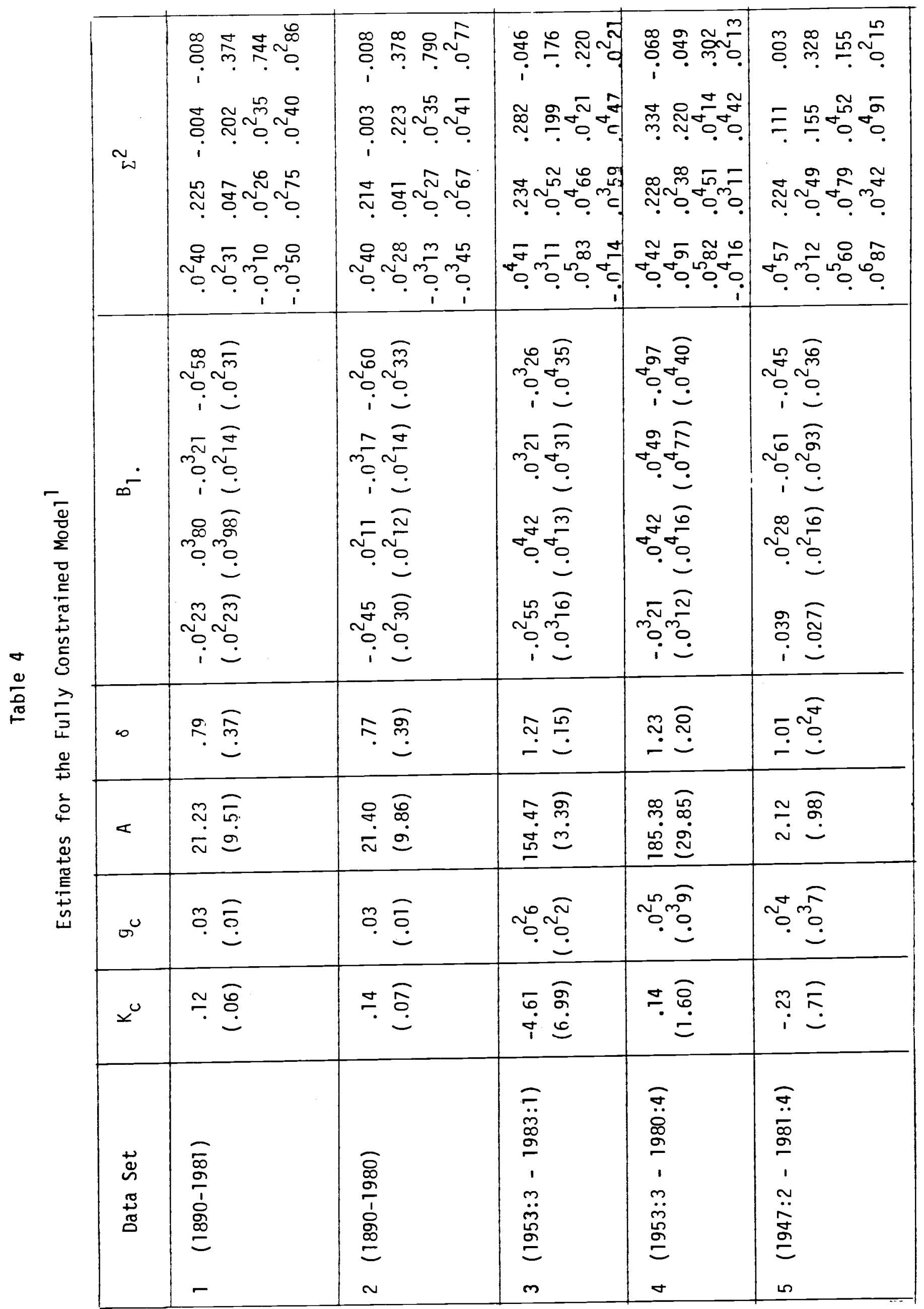




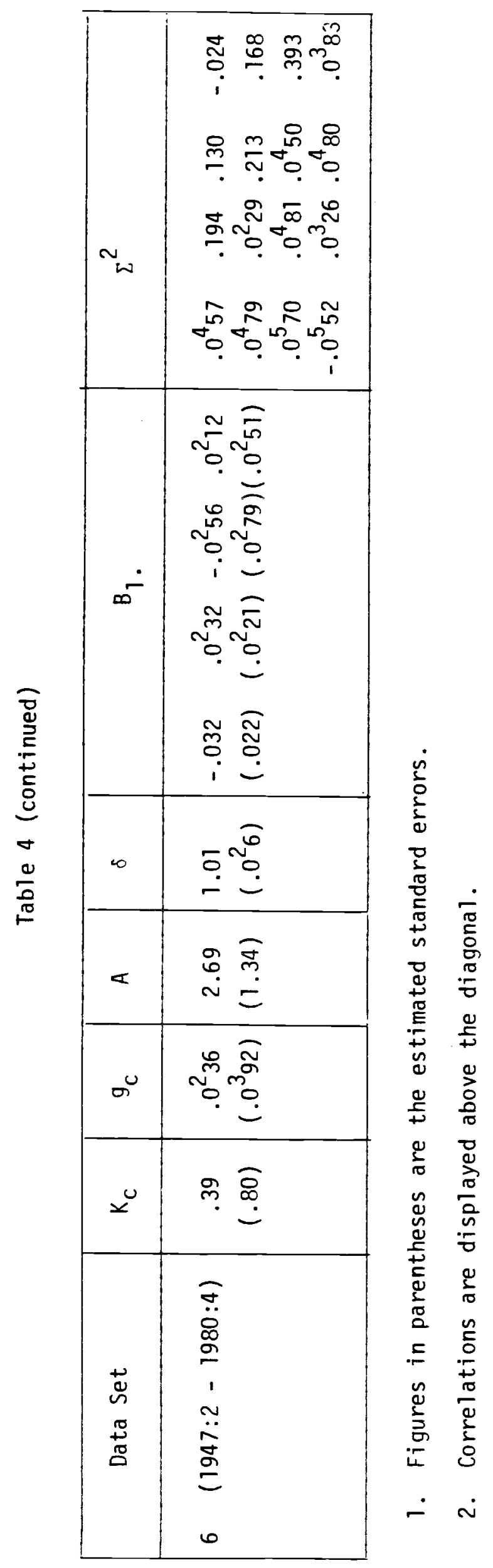




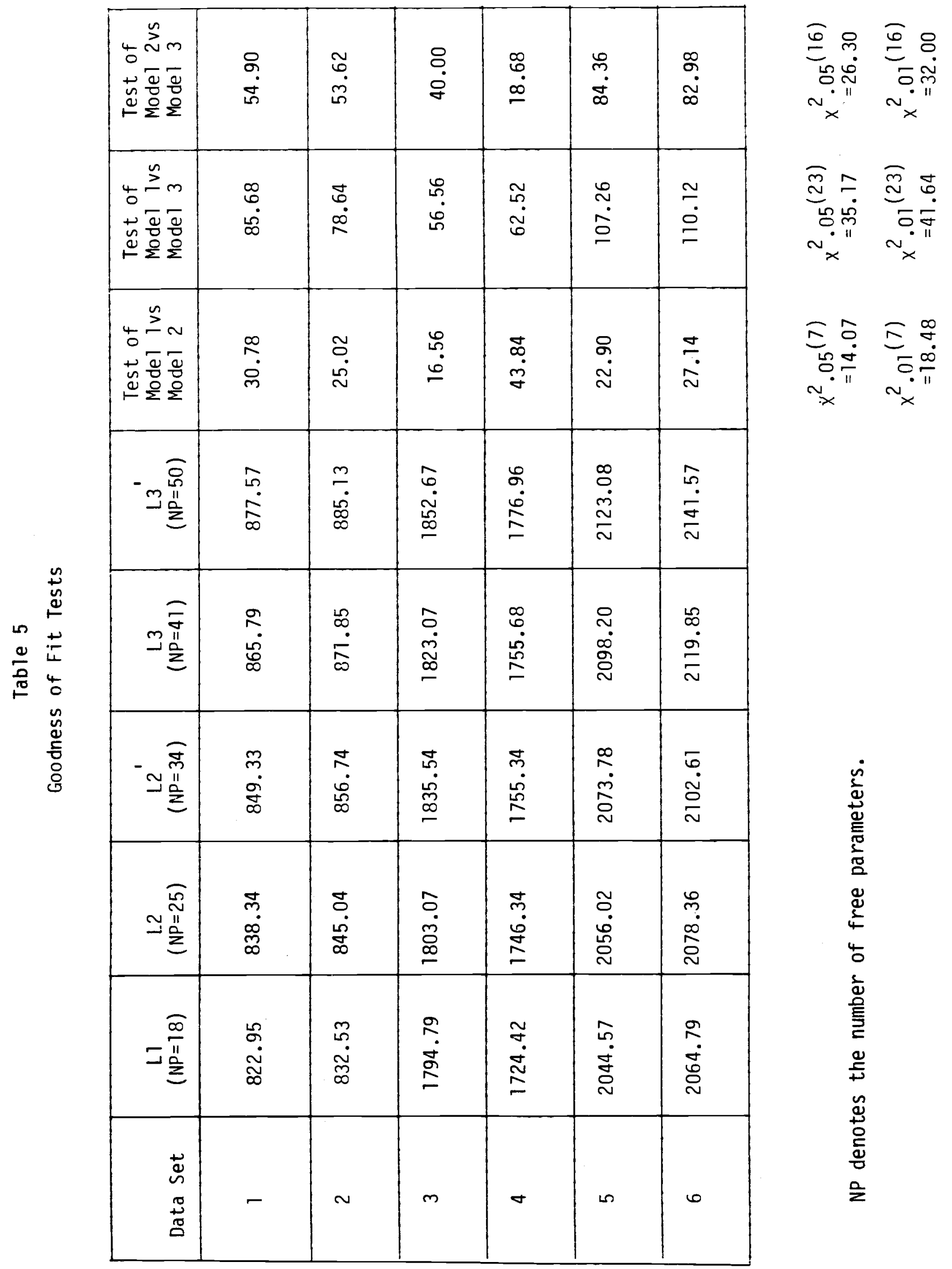




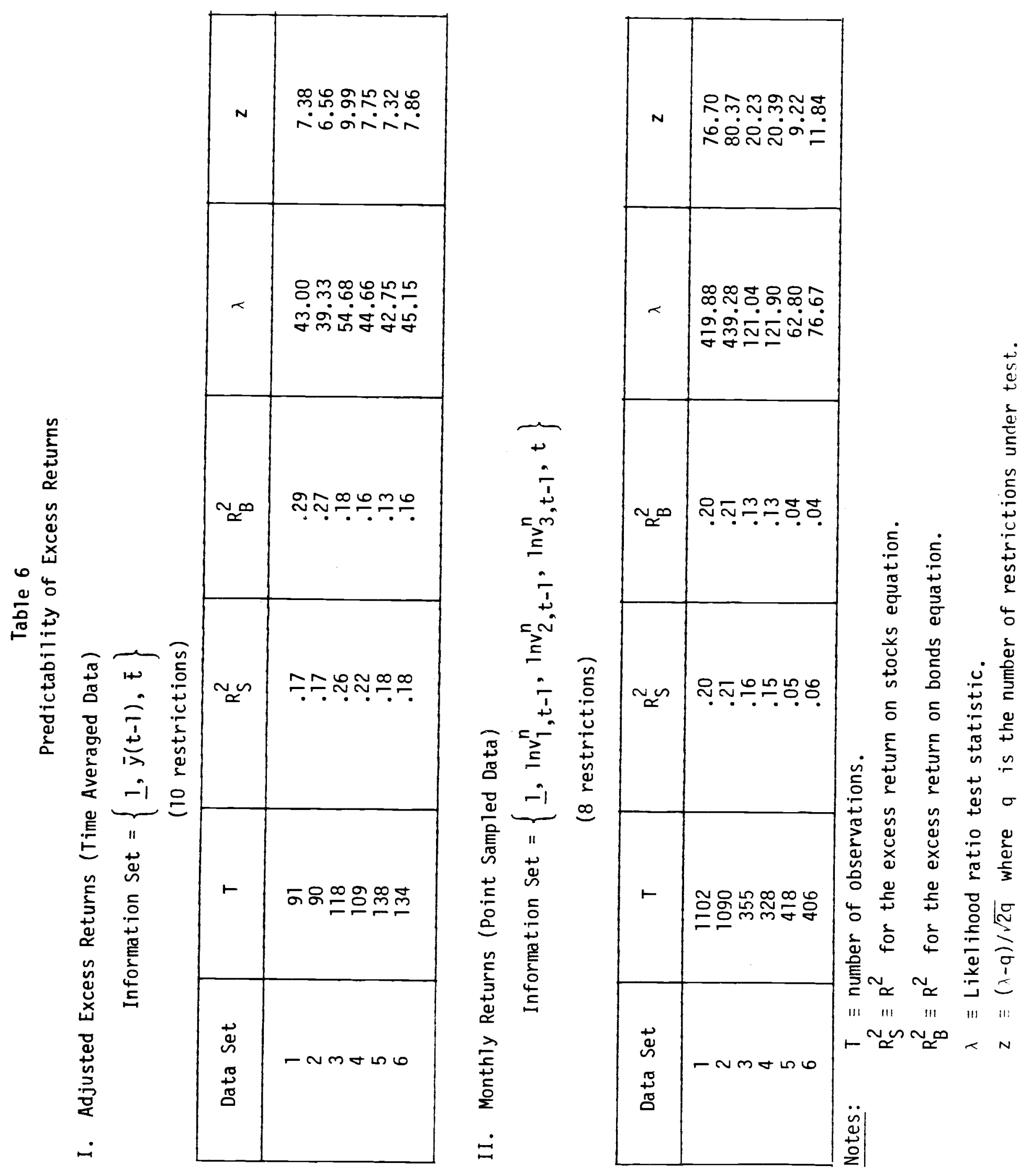




\section{Fieferences}

Eergetrom,A.F.' 'Continuous Time Stochastic Models and Issues

of Aggregation Over Time,' in Z. Griliches and M. Intriligator (eds) Handboot: of Econometrics, Amsterdam: North-Hol 1 and, (1984)

Ereeden,D.T. 'An Intertemporal Asset Fricing Model with Stochastic

Consumption and Investment Opportunities,' Journal of Financial

Economics, $7(1979): 265-296$

Grariger, C.W.J. and Fi.F. Engle 'Dynamic Model Specification with

Equilitrium Constraints: Co-integration and Error Correction,

Department of Economics W.P.\#85-18, University of California,

San Diego, revised May 1985

Grossman, S.J. and F.J. Shiller 'Freliminary Fiesults on the

Determinarits of Stock: Martiet Variability,' unpublished mimeo, June 1980

-'On the Determinants of Stock Market Variability,' American Economic Feview, 7(1981):222-227

-'Consumption Correlatedness and Fist: Measurement in Economies with Non-traded Assets and Heterogeneous Information,' Journal of Financial Economics,10(1982):195-210

Hannan,E.J. Multiple Time Series, New York:Wiley,1970

Hannan,E.J., W.T.M. Dunsmuir and M. Deistler 'Estimation of Vector AFimax Models,' Journal of Multivariate Analysis, $10(1980): 275-295$

Hansen,L.F'. and K.J. Singleton 'Stochastic Consumption,Fisk: Aversion, and the Temporal Behaviour of Asset Feturns,' Journal of Folitical Economy, $91(1983): 249-265$ 
Ibbotson,Fi.G. and F.A. Sinquefield Stoctss, Bonds, Bills and Inflation: The Fist and the Future,

Charlottesville: Financial Analysts Fiesearch Foundation, 1982 Melino,A. 'Estimation of Unit Averaged Diffusion Frocesses,'

Departmerit of Economics-Institute for Folicy Analysis

W. F'. \#8502, University of Toronto, March 1985

Merton,Fi.C. 'Optimum Consumption and Fortfolio Filles in a Continuous

Time Model,' Journal of Economic Theory, $\$(1971): 375-413$

Osborne,D.Fi. 'Exact and Approximate Maximum Lil:elihood Estimators

for Vector Moving Average Frocesses,' Journal of the Foyal

Statistical Society, Series $\mathrm{B}, \quad 39(1977): 114-118$

Fhillips,F'.C.B. 'The Treatment of Flow Data in the Estimation

of Continuous Time Systems,' in Eergstrom et.al (eds), Stability and Inflation, New York: Wiley, 1978

Seltzer, L.H. The Nature and Tax Treatment of Capital Gains and

Losses, New York: National Bureau of Economic Fiesearch,1951

Shiller,Fi.J. 'Consumption, Asset Markets and Macroeconomic

Fluctuations,' Carnegie-Fochester Conference Series, 17 (1982)

Wilson,G.T. 'The Factorization of Matricial Spectral Densities,'

SIAM Journal of Applied Mathematics, 23(1972):420-426

- The Estimation of Farameters in Multivariate Time Series Models,' Journal of the Foyal Statistical Society, Series B, $35(1973): 76-85$ 